\title{
A C-Band Study of the Historical Supernovae in M83 with the Karl G. Jansky Very Large Array
}

\author{
Christopher J. Stockdale ${ }^{1}$ \\ Marquette University
}

Knox S. Long ${ }^{2}$

Space Telescope Science Institute

Roberto Soria

Curtin University

John J. Cowan

University of Oklahoma

Larry A. Maddox

Northrop Grumman

P. Frank Winkler

Middlebury College

Kip D. Kuntz

Johns Hopkins University

William P. Blair

Johns Hopkins University

\author{
James Miller-Jones \\ Curtin University
}

\begin{abstract}
We present new low frequency observations of the grand design spiral galaxy, M83, using the C and L bands of the Karl G. Jansky Very Large Array (VLA). Utilizing the newly expanded bandwidth of the VLA, we are exploring the radio spectral properties of the more than 150 radio point sources in M83. We present the initial analyses, focusing on the radio evolution of the six historical supernovae discovered in the last century. With four epochs of VLA observations and optical and X-ray observations, we will probe the transition of supernovae into supernova remnants.
\end{abstract}

${ }^{1}$ CJS thanks the Wisconsin Space Grant Consortium, the Distinguished Visitor Programs of the Australian Astronomical Observatory and NRAO, the University of Oklahoma, and Marquette University for their financial support of this research program.

2 KSL acknowledges Chandra Award Nos. G01-12115A, B, and C issued by the CXC, which is administered by SAO, and HST grant GO 12513, provided by NASA through the STScI, which is operated by AURA, Inc., under contract NAS5-26555 


\section{Introduction and Motivation}

As part of a long-term study to detect radio emission from known (i.e., historical) supernovae (SNe), the Karl G. Jansky Very Large Array (VLA) ${ }^{3}$ has been used to observe a number of galaxies (Cowan \& Branch 1985; Cowan, Goss \& Sramek 1991; Cowan, Roberts \& Branch 1994; Eck et al. 1998; Eck, Cowan \& Branch 2000; Stockdale et al. 2001; Stockdale et al. 2006; Maddox et al. 2006). The primary goal of these observations has been to learn more about the evolution of SNe into supernova remnants (SNRs), how radio emission is produced in these events, and the environment in which they occur. In the course of this effort, we have made very deep, arcsecond resolution observations of the nearby spiral galaxy, M83, and we have obtained deep, similar resolution measurements at X-ray and optical wavelengths which have allowed us to examine the point source populations of this galaxy, discovering a number of previously unreported HII regions, SNRs, X-ray binaries, and Ultra-Luminous X-ray sources. In the last 100 years, six optical SNe have been detected in M83. Radio emission has been detected from four of these recent SNe, all but SN 1945B (unknown optical type, possible a type Ia SN) and SN 1968L (buried in the bright but complex nuclear region). In recent Hubble Space Telescope (HST) observations, SN 1968L was optically recovered (Dopita et al. 2010). Given this recent SN activity, we expect to find $\sim 120$ SNRs that were formed in the last 2,000 yrs. This makes M83 one of the closest $\mathrm{SN}$ factories and the ideal galaxy to study young SNRs.

For this project, we made new, deep, VLA observations of M83 at $5.0 \mathrm{GHz}$ and $1.4 \mathrm{GHz}$ utilizing the broad spectral coverage of the VLA. We

- searched for "hidden" SNRs in the extensive HII continuum emission of the spiral arms;

- monitored the time evolution of historical SNe in M83 as they transition into SNRs;

- explored the spectral profile of the nuclear region of M83; and

- studied the spectral properties of the diffuse emission in the spiral arms in conjunction with new HST and Chandra studies.

These new measurements were $10 \times$ deeper at every sub-band than any previous VLA observations of M83. Figure 1 illustrates an initial analysis of the new Australia Telescope Compact Array radio data in the $\mathrm{C}$ band and $160 \mathrm{ks}$ of recent Chandra $(160 \mathrm{Ks}) \mathrm{X}$-ray observations of M83 and what we can begin to expect from deeper imaging of the galaxy with a total of $750 \mathrm{ks}$ of Chandra new observations.

3 The Karl G. Jansky Very Large Array telescope managed by the National Radio Astronomy Observatory which is a facility of the National Science Foundation operated under cooperative agreement by Associated Universities, Inc. 


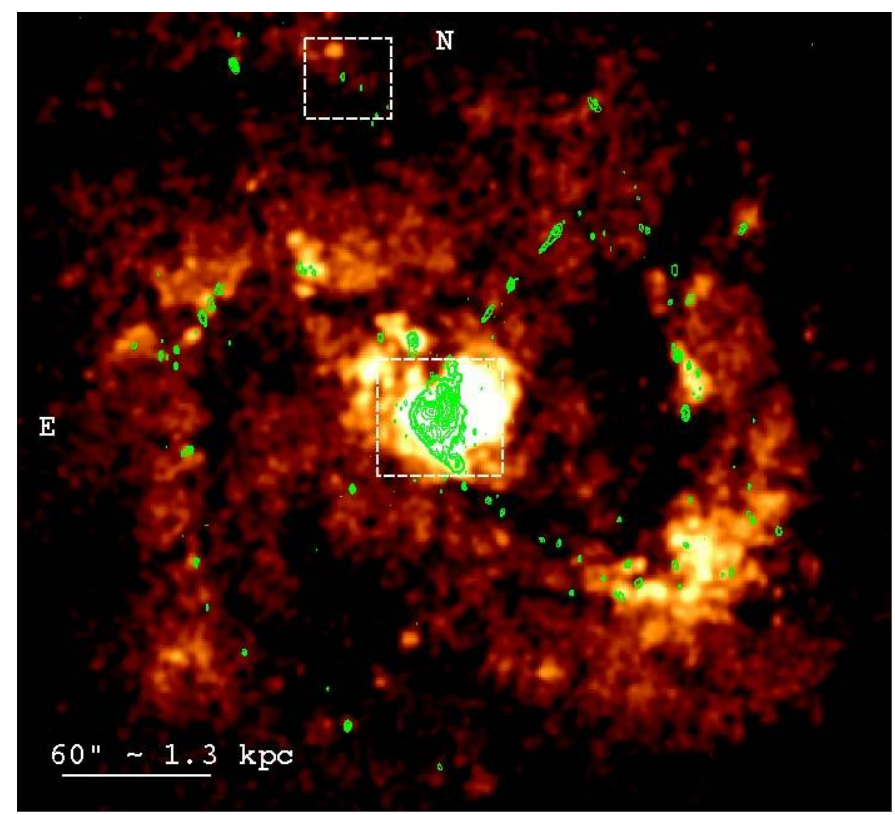

Figure 1: The C-band ATCA radio contours image of M83 overlaid on the diffuse X-ray emission, from our initial Chandra observations of $160 \mathrm{ks}$ (.3-7 $\mathrm{keV}$ ), provide a tantalizing glimpse of what we can expect from the complete $750 \mathrm{ks}$ dataset. The two boxes identify the regions more fully explored in Figure 3.

Previous studies of M83 by Maddox et al. (2006) identified a number of radio point sources with $\mathrm{C}$ and $\mathrm{L}$ bands pseudo-continuum measurements that had radio spectral indices $\left(\alpha, \mathrm{S}_{v} \sim v^{\alpha}\right)$ that were neither clearly thermal nor non-thermal. This was likely caused by spatially coincident HII regions and SNRs with blended emission, causing the flux from thermal HII regions to dominate the higher frequency emission and the SNR flux to dominate at lower frequencies. This made it difficult to definitively identify a possibly significant portion of the SNRs that are in the galaxy. In 2011, we proposed a scaled-array experiment to measure the spectral index evolution of a sample roughly double the size of the $\sim 50$ sources that were detected by Maddox et al. (2006), as they vary across the EVLA L and C Bands. This project gives comparable resolution at both $\mathrm{C}$ and $\mathrm{L}$ band, and allows us to image each sub-band separately, to contrast the full spectral profiles of each source detected in the two EVLA bands. With these images, we are able to spectroscopically distinguish the non-thermal emission from the SNRs from the thermal emission associated with the large, diffuse HII regions. The non-thermal emission from these hidden SNRs should become a more significant part of the radio emission especially at the lower frequencies of the $\mathrm{L}$ band.

This radio project is part of a large multiwavelength study of M83, the nearest face on, granddesign spiral galaxy with a starburst, and the site of 6 historical SNe. The study includes deep Xray imaging with Chandra (750 ks PI Long) and optical imaging with Magellan (PI Winkler) and HST observations (PI Blair; Dopita et al. 2010). Radio observations are crucial for resolving the nature of the source populations, especially SNRs seen in the X-ray and optical surveys, with 71 optically identified SNR candidates and with 20-30 X-ray counterparts (Blair \& Long 2004). Consequently, we have made new ATCA observations at S and C bands to attain higher sensitivity at these wavelengths than was possible with our earlier VLA observations (Figure 1). Well determined radio spectral indices and X-ray colors make it possible to clearly identify the precise nature of sources in a given galaxy (Maddox et al. 2006; Kilgard et al. 2005; and Prestwich et al. 2003). 


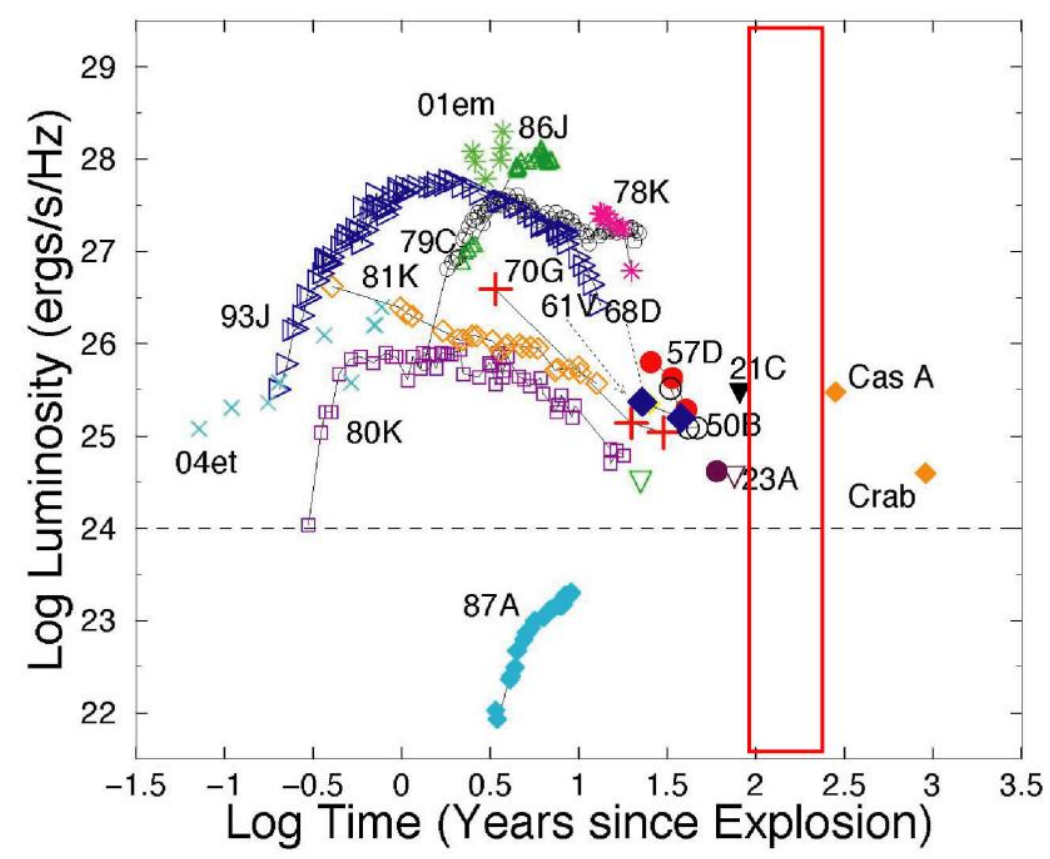

Figure 2.- Radio light curves for historical type II SNe in M83 at L band compared to several radio SNe II and SNRs. [SNe 1923A (filled circle and open, inverted triangle for upper limit), 1950B (open circles) \& 1957D (filled circles, and each as labeled in the figure.] (Stockdale et al. 2006). The red rectangle represents the region where we expect these supernovae to transition into supernova remnants in the 100-300 years following explosion.

\section{Past Observations of M83}

Our collaboration has studied the long term radio emission from compact sources in the galaxy M83 since the commissioning of the VLA in the early 1980's. The deep continuum VLA observations span a period of approximately 15 years, from 1982 to 1998. In Maddox et al. (2006), we presented the data over the fifteen years of VLA monitoring with a consistent data analysis and imaging scheme to ensure the best possible comparative study of more than 50 sources and extensive diffuse emission regions across the galaxy. Some of our key findings include:

- It was shown that SN 1957D has continually faded in the radio from 1982-1998, consistent with a shock expanding through a circumstellar material that is decreasing in density (Figure 2). SN 1923A has faded to near the limits of detection of these observations. We continue to show no detection of SN $1983 \mathrm{~N}$ after its initial radio detection, consistent with it having been a Type Ib supernova. SN 1950B has apparently faded to the level of thermal HII regions that are near the position of the explosion. SN 1923A has faded to near the limits of detection of these observations. Figure 2 illustrates the $\mathrm{L}$ band radio evolution of a variety of decades-old SNe.

- About half of the radio sources are thermal HII regions, $\alpha \sim 0.0$. The HII regions tend to be very large, exhibiting high excitation parameters. The largest regions were not detected due to the high resolution of the previous VLA observations.

- It was found that ten sources were coincident with X-ray sources. The continuum spectral indices of these sources indicated that most were X-ray supernova remnants. We 
confirmed that one of the coincident sources (source 28) is the nucleus of a background radio galaxy with two radio lobes (sources 27 and 29). Three of the X-ray sources are coincident with known optical supernova remnants.

\section{Why We Needed New Observations of M83}

Studies of the compact sources are providing new information about late-term stellar evolution (e.g.,mass-loss rates), emission mechanisms, the transition of SNe into SNRs, and the nature of XRBs. Radio, X-ray, and optical observations provide three independent and complementary means to identify SNRs - a non-thermal radio spectrum, soft X-ray spectra (with line emission if there are sufficient counts) and emission line ratios showing shocked gas. Only through their combination can we carry out a complete census of SNRs in M83 and begin to understand the environmental conditions that lead to their identification in different wavelength ranges.

With these new observations, we are exploring the spectral properties of the diffuse emitting regions along the spiral arms and in the nuclear of M83 to constrain to the extent that various emission mechanisms contribute. The soft X-ray diffuse emission in the spiral arms of M83 is the brightest of any known spiral galaxy (see Figure 1).

We note that the nuclear region of M83 (Figure 3, right) has shown a slight increase in 1998 VLA L band emission in the radio peak, not seen in the $\mathrm{C}$ band data. It is possible that there is an increase in accretion onto a supermassive black hole, which would be consistent with X-ray results. The reported optical/IR nuclear peak is not consistent with the brightest radio nucleus, though there is evidence for a radio emission region at the position of the optical nucleus. The nuclear radio peak is near the position of a second "dark" nuclear mass concentration that corresponds to the dynamical nucleus of the galaxy, possibly a double massive black hole system. Our spectral index observations will allow us to search for possible jet emission to help confirm this hypothesis.

With these new EVLA observations (with similar BW coverage and configurations as the previous three epochs of VLA studies), we are able to follow the long-term evolution of all of these sources in M83 in comparison with more than 25 years of previous VLA and Chandra studies. We would like to note some initial discoveries made in the initial Chandra observations (December 2010). These new X-ray images reveal significantly more sources than in the earlier (shorter) observation, including a previously undiscovered ULX source and significant change in the X-ray emission of SN 1957D (Figure 3, left). The X-ray emission associated with SN 1957D has brightened significantly, never having been detected in earlier epochs. This could indicate the formation of a pulsar wind nebula, a significant change in the density of the circumstellar wind established by the stellar wind of the progenitor star, or the SN blastwave is now encountering significant amounts of ISM and completing its transition into a shell SNR. With so many older historical SNe, M83 is the best galaxy to study the SN to SNR transition.

Recently, Chomiuk \& Wilcots (2009) have argued that radio SNRs have a universal luminosity function, with a normalization proportional to the star formation rate in a galaxy but independent of ISM density. M83 is the ideal galaxy to fully explore the history of star forming rate (SFR) of a nearby spiral and to test their model, we need large, well-studied samples in nearby galaxies. 
From the initial VLA maps, Maddox et al. (2006) found 17 candidate radio SNRs, about half of which are also detected at optical and/or X-ray wavelengths. With this project, we will use previously detected VLA sources like SN1950B and other marginally radio-identified SNRs to explore their spectral index across both bands and apply what we learn to newly discovered sources to more clearly identify as SNRs or not with the VLA.

Assuming the Chomiuk \& Wilcots (2009) model is correct, we predicted that we would find 100 candidate radio SNRs with our ATCA/EVLA observations, including confirming the identification of a number of marginally detected SNRS in the Maddox et al. (2006) survey. Finding these SNRs is important to verify or refute the Chomiuk \& Wilcots (2009) model, as well as for helping to determine the nature of the approximately 600 point sources expected in the new Chandra survey of M83. The full analysis of these VLA measurements will provide crucial evidence to fully explore M83's star formation history.
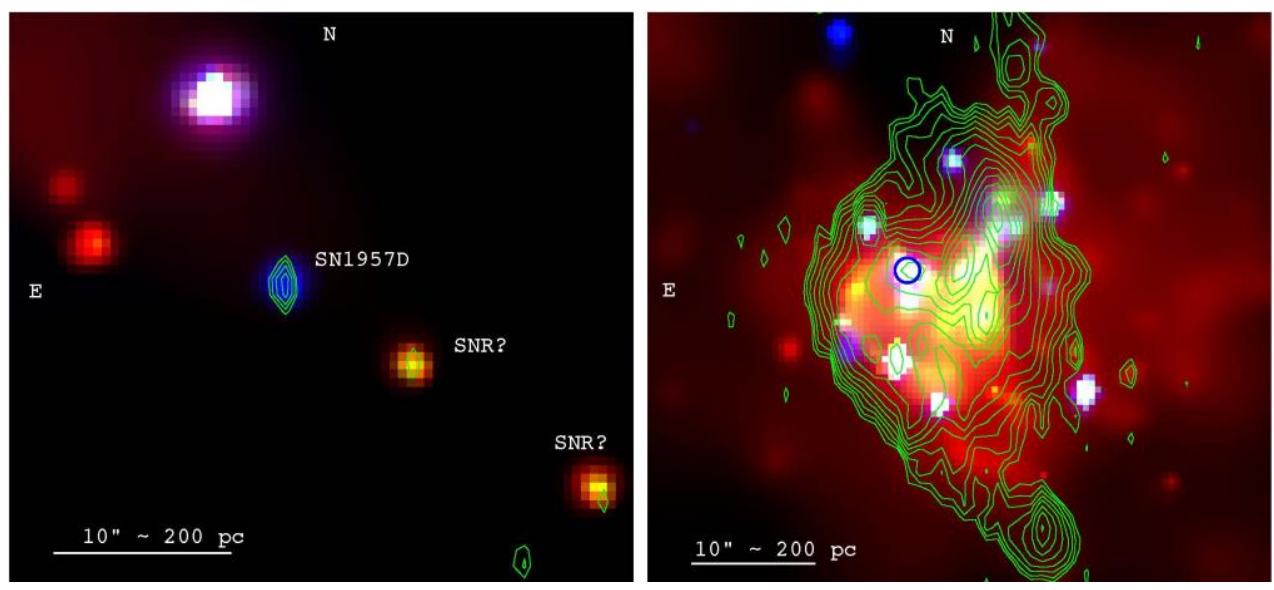

Figure 3.- ATCA, C-band, Radio contours image of M83 overlaid on the diffuse X-ray emission. Left: The Xray/radio detection of SN 1957D with two SNR candidates along the northern spiral arm. Right: The radio contours indicate 4 central radio sources with the brightest significantly offset from the optical peak (as labeled in left figure).

\section{Low Frequency Radio Spectra of Historical Supernovae}

We obtained 14 hours of $\mathrm{C}$ band observations of M83 in the $\mathrm{CnB}$ configuration sampling the $4 \mathrm{GHz}$ bandwidth. We also obtained 16.0 hours of $\mathrm{L}$ band observations of M83 in the BnA configuration sampling a $1 \mathrm{GHz}$ bandwidth. With these observations, we achieved an average 3 sigma detection threshold at $\mathrm{L}$ band of $30 \mu \mathrm{Jy} / \mathrm{bm}$ and at $\mathrm{C}$ band of $21 \mu \mathrm{Jy} / \mathrm{bm}$. We obtained a somewhat elongated beam, $4 \times 3 \operatorname{arcsec}^{2}$ or $80 \times 60 \mathrm{pc}$, for the central sub-band, which will varied across both bands, and we wereable to adjust the beam size to match resolution of the prior VLA observations of M83. Each arcsec corresponds to $20 \mathrm{pc}$ at $4.5 \mathrm{Mpc}$. The data were analyzed using the CASA software provided by the National Radio Astronomy Observatory (NRAO). We followed scripts for low frequency data analysis prepared by Miriam Krauss, NRAO Jansky Postdoctoral Fellow, and posted on the www.nrao.edu website. Due to significant radio frequency interference (RFI) in the L band data, we were forced to discard, or flag, roughly one third of the data that was unable to be calibrated. Here we present the preliminary analysis 
of our observations of the recent VLA data regarding the historical supernovae located in M83 (see Figure 4).

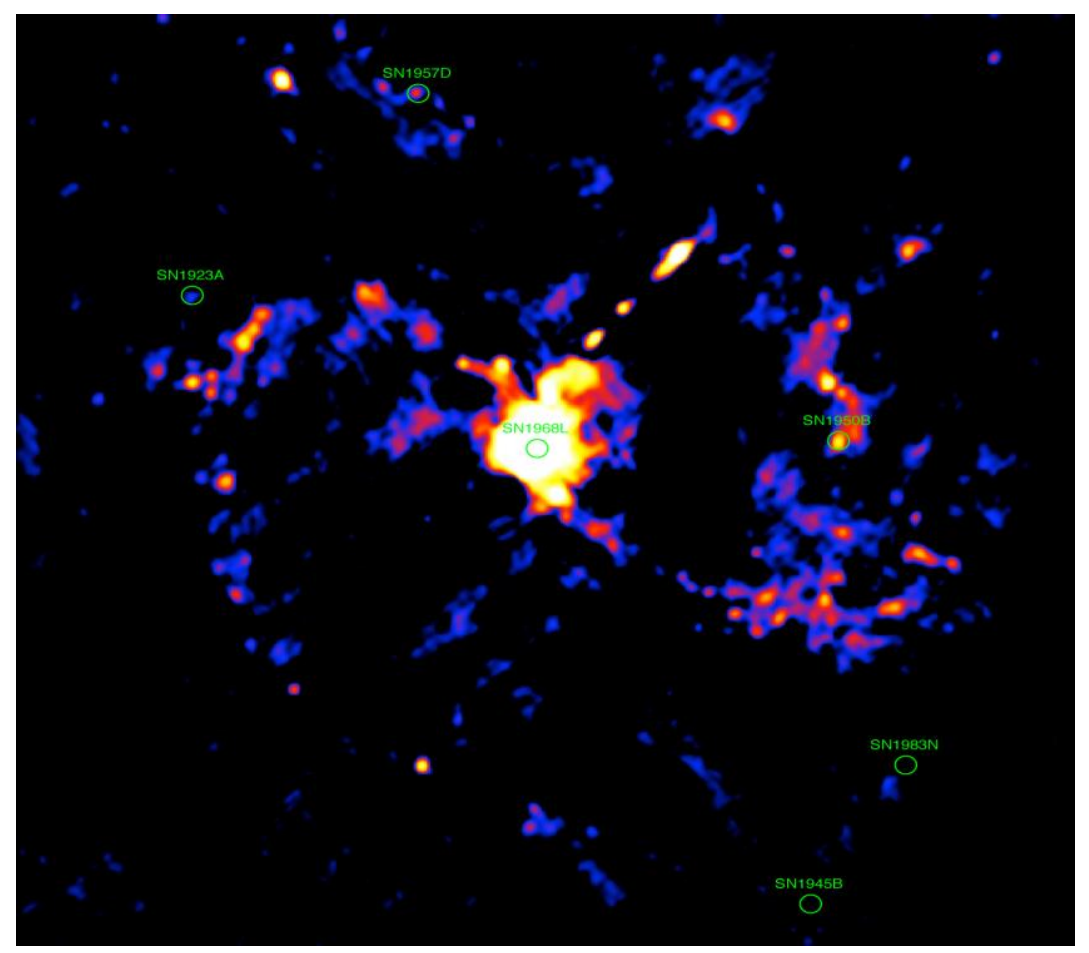

Figure 4. L band radio image $(1 \mathrm{GHz}$ ) centered on $1.5 \mathrm{GHz}$ with the optically reported positions of the six historical supernovae indicated. Rms noise for the image is 30 microJy. The diffuse emission associated with the radio nucleus has two distinct radio peaks which are coincident with the optical and X-ray central sources detected with HST and Chandra).

In these recent VLA observations, we detect radio emission from the vicinity of 3 of the 6 historical SNe in M83: SN 19757D, SN 1950B, and SN 1923A. The C Band radio emission appears to be dominated by thermal Bremsstrahlung associated with nearby or associated HII regions for all three detected sources. The L Band radio emission for SNe 1957D and 1923A appears to have a significant non-thermal component, attributed to synchrotron emission from either the SN shock interacting with circumstellar and/or interstellar medium or other nearby, older SN remnants (See Figure 4). 
Historical Supernovae in M83

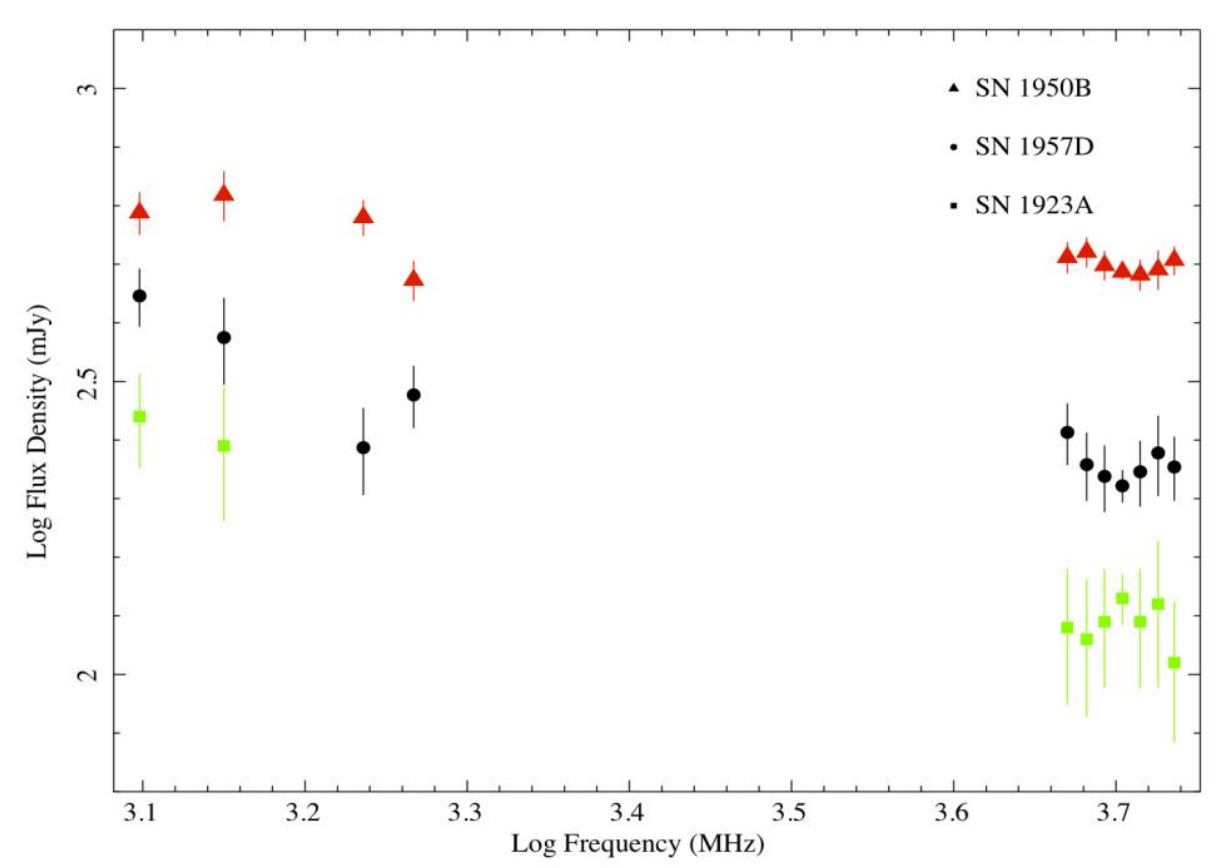

Figure 5. The log of flux densities for the three historical supernovae are plotted above as a function of log frequency. The $\mathrm{C}$ band measurements at right indicate thermal emission. The $\mathrm{L}$ band measurements at left appear to indicate non-thermal emission as the flux increases at lower frequencies for SNe 1957D and 1923A.

In Figure 5, we plot the new radio VLA data vs frequency and there appears to be a clear steepening of the radio emission from SNe 1957D and 1923A, helping to confirm our detection of emission from the supernova shocks interacting with the material ejected in the winds of the their progenitor stars prior to explosion.

We also detect approximately 130 point individual point sources have been detected. It is our intent to perform a spectral analysis of these sources to search for older, "hidden" supernova remnants who's radio emission is often dominated at higher frequencies by thermal emission from nearby HII regions. It is our intent to expand this analysis to other nearby galaxies and further improve our understanding of how stars form in nearby in spiral galaxies. 


\section{References}

Blair \& Long, 2004, Astrophysical Journal Supplement, vol. 155, p. 101

Chomiuk \& Wilcots, 2009, Astrophysical Journal, vol. 703, p. 370

Cowan \& Branch, 1985, Astrophysical Journal, vol. 293, p. 400

Cowan, Goss, \& Sramek, 1991, Astrophysical Journal, vol. 379, p. L49

Cowan, Roberts, \& Branch, 1994, Astrophysical Journal, vol. 434, p. 128

Dopita, Blair, Long, Mutchler, Whitmore, Kuntz, Balice, Bond, Calzetti, Carollo, Disney,

Frogel, O’Connell Hall, Holtzman, Kimble, MacKenty, McCarthy, Paresce, Saha, Silk, Sirianni,

Trauger, Walker, Windhorst, \& Young 2010, Astrophysical Journal, vol. 710, p. 964

Eck, Cowan, \& Branch, 2002, Astrophysical Journal, vol. 573, p. 306

Eck, Roberts, Cowan, \& Branch, 1998, Astrophysical Journal, vol. 508, p. 664

Kilgard, Cowan, Garcia, Kaaret, Krauss, McDowell, Prestwich, Primini, Stockdale, Trinchieri, Ward, \& Zezas 2005, Astrophysical Journal Supplement, vol. 159, p. 214

Maddox, Cowan, Kilgard, Lacey, Prestwich, Stockdale, \& Wolfing, 2006, Astronomical Journal, vol. 132, p. 310

Prestwich, Irwin, Kilgard, Krauss, Zezas, Primini, Kaaret, \& Boroson, 2003, Astrophysical Journal, vol. 595, p. 719

Stockdale Goss, Cowan, \& Sramek, 2001, Astrophysical Journal, vol. 559, p. L139

Stockdale, Maddox, Cowan, Prestwich, Kilgard, \& Immler, 2006, Astronomical Journal, vol. 131, p. 889 